\title{
Gisela Trommsdorff
}

\section{Internationale Kultur?}

\section{Kulturpsychologische Aspekte der Globalisierung}

Die weltweite Vernetzung individueller und nationaler Interessen und Kontakte, die internationale Verbreitung von Informationen, Verflechtung von Wirtschaft und Politik, die hier als Aspekte von Globalisierung verstanden werden, oder die Migrantenströme in verschiedensten Teilen der Welt sind unübersehbare Aspekte einer zunehmenden Internationalisierung von Personen und Institutionen. Diese Internationalisierung ist unaufhaltsam und stellt neue Anforderungen an den einzelnen und die Gesellschaft. Eine Abriegelung von dieser Internationalisierung und weltweiten Verflechtung läßt sich heute kaum realisieren; das gilt selbst für totalitäre Gesellschaften. Dies bedeutet, dass unausweichlich ist, sich mit den kulturellen Besonderheiten von Menschen und Institutionen in anderen Gesellschaften auseinanderzusetzen. Dies ist in besonderem Maße erforderlich, wenn das Ziel besteht, lokale Märkte global zu erweitern oder in international zusammengesetzten Gruppen erfolgreich zu kooperieren, weil damit Begegnungen mit anderen Kulturen verbunden sind. Solche Kulturbegegnungen führen irgendwann zu der Erfahrung, dass es wichtig ist, die Kulturbesonderheiten, also die andere Sprache, andere Verhaltensregeln und Werte zu kennen und sich angemessen darauf einzustellen. Dies ist jedoch schwierig und häufig die Voraussetzung von Mißverständnissen und Konflikten.

Dass durch Kulturbegegnungen solche Kulturunterschiede vermindert oder vielleicht verstärkt werden, ist eine Frage, die in dem vorliegenden Beitrag eine wichtige Rolle spielt. Im folgenden soll der Frage nachgegangen werden, ob die zunehmenden globalen Verflechtungen zur Entstehung einer globalen, internationalen Kultur führen werden, in der lokalen Kulturbesonderheiten keine Bedeutung mehr zukommt. Zunächst sei kurz etwas zu dem Kulturbegriff und möglichen Folgen von Kulturbegegnungen gesagt, um dann vier Thesen sowie eine weitere psychologische These zu diskutieren.

\section{I.}

\section{Kulturen als komplexe und sich wandelnde Kontexte}

Nachdem sich die Anthropologie von dem Kulturbegriff verabschiedet hat, ist der Kulturbegriff aus den Sozialwissenschaften nicht mehr wegzudenken, und er ist im Alltag in aller Munde - angefangen von der ,multikulturellen Gesellschaft" über „Jugendkultur“", ,Gegenkultur“, „Unternehmenskultur“ bis hin zu über Internet geschaffene Kulturen der virtuellen Welt. Aus wissenschafts- und forschungspolitischer Sicht erscheint es inzwischen höchst bedeutsam, die 
Geisteswissenschaften „kulturwissenschaftlich“ zu verändern. Der Kulturbegritt ist unscharf. Es gibt weit über hundert Definitionsversuche, und es erfolgen immer noch weitere Definitionen von Kultur. So ist die Versuchung groß, schwer zu beschreibende komplexe Phänomene mit einem anspruchsvollen Begriff zu versehen, und das "label“ mit der Erklärung des Phänomens zu verwechseln. Läßt sich die gegenwärtig bestehende Inflation des Kulturbegriffs als eine Folge der Wahrnehmung von Kulturunterschieden und der Erfahrung von Kulturbegegnungen sehen und als eine Folge von Konflikten zwischen Vereinheitlichungs- und Individualisierungsdruck?

Betrachten wir zunächst, wie Kulturen entstehen, um dann zur Frage einer empirischen Beschreibung von Kulturen zu kommen.

1 . Zur Entstehung von Kultur

Das Entstehen von Kulturen läßt sich erklären als koordiniertes Verhalten zur gemeinsamen Lösung von Überlebensproblemen und damit zur gemeinsamen Überwindung von natürlichen Gefährdungen bzw. Nutzbarmachung natürlicher Ressourcen. So können kulturelle Ressourcen aufgebaut werden. Die klimatischen und ökologischen Bedingungen werden häufig als wichtige Voraussetzung für die Entstehung von Kulturen gesehen (vgl. Whiting \& Whiting 1975). Die in der Gruppe erworbenen, vermittelten und geteilten Kenntnisse über Techniken des Überlebens sowie des erfolgreichen Zusammenlebens stellen kulturelles Wissen dar, das unter bestimmten Bedingungen an die nächste Generation weitergegeben wird. Die Weitergabe dieses Wissens setzt eine gemeinsame Sprache sowie geteilte Überzeugungen hinsichtlich der Bedeutung bestimmter objektivierbarer Phänomene (Natur, Sprache, Artefakte etc.) und die Akzeptanz von Vermittlungsagenten (Gruppenangehörige, Häuptling, Verwandte, Ältere) voraus. Damit werden Voraussetzungen für Gemeinsamkeiten in Handeln, Denken, in der Sprache, im Umgang mit Problemen sowie für die Strukturierung der Gruppe und für die Wahrnehmung des Selbst entwickelt. Es läßt sich annehmen, dass Kultur die Grundlage für die Entstehung von sozialen Interaktionen (z.B. bestimmten Gemeinschaften) für die Herausbildung von individuellen Fähigkeiten und Zielen ist, wobei hier Wechselwirkungen bestehen: Soziale Interaktionen (und Systeme) beeinflussen die Kultur, und individuelles Handeln beeinflußt soziale Systeme und kulturelle Besonderheiten. Kultur kann somit durch soziale Interaktion und individuelles Handeln hergestellt, stabilisiert und verändert werden. Ressourcen für Kultur gehen diesen sozialen und individuellen Konstruktionen jedoch aufgrund historisch vermittelter Wechselwirkungen zwischen natürlichen Umwelten und menschlichen Anpassungsleistungen voraus. 
Aus psvchologischer Sicht kann die Entwicklung individueller Persönlichkeiten einschließlich Fähigkeiten und Ziele nicht unabhängig von den kulturellen Bedingungen gesehen werden, unter denen Menschen aufwachsen. Dabei werden soziale Systeme und Sozialisationsbedingungen als vermittelnde Prozesse verstanden (Trommsdorff 1998c). Identitätsentwicklung ist z.B. immer eingebunden in Werte und Deutungssysteme der jeweiligen Kultur, in dèr die Person aufwächst, und wird vermittelt durch die Zugehörigkeit zu einer Gruppe. die durch gemeinsame Ziele und Werte und bestimmte soziale Beziehungen definiert ist. Diese Auffassung läßt sich gut mit dem Ansatz von Super \& Harkness (1986) verbinden, wonach die individuelle Entwicklung in ,cultural niches" erfolgt, d.h. die Persönlichkeitsentwicklung wird von kulturell vermittelten Werten, Erziehungsvorstellungen der Eltern, Erziehungsmethoden etc. beeinflußt. Diese Auffassung ist auch mit dem nunmehr klassischen Ansatz von Bronfenbrenner (1979) zur ökologischen Bedingtheit von Entwicklung vereinbar und legt ein Mehrebenenmodell für die Analyse des Zusammenwirkens ökologischer. sozialer und psychologischer Bedingungen für die Rolle der Kultur in der menschlichen Entwicklung nahe.

Wie lassen sich Kulturen empirisch beschreiben?

Zur Individualismus-Kollektivismus-Debatte

Je nachdem, ob man von Jugend- und Lebensstilkulturen ausgeht oder ethnische Gruppen oder gar Nationen oder Sprachgemeinschaften als Einheiten für die Abgrenzung von „Kulturen“" wählt, sind ganz unterschiedliche Phänomene zu beschreiben. Bei der Beschreibung von Kulturen besteht zum einen das Problem, abgrenzbare Einheiten von Kulturen zu bestimmen. Solche Einheiten lassen sich nicht unbedingt an formalen Kriterien wie nationalen Grenzen festlegen. Wegen der Willkürlichkeit der häufig historisch bedingten staatlichen Grenzziehung sind Nationen keineswegs immer geeignet, Kulturbesonderheiten abzugrenzen, wie die vielen Konfliktherde, z.B. in Afrika zwischen den Hutu und Tutsi, zwischen den Iren und Engländern oder zwischen den Serben, Kroaten und Slowenen im ehemaligen Jugoslawien deutlich machen.

Zum anderen besteht das Problem, Kulturen in ihren Besonderheiten inhaltlich zu beschreiben. Aus psychologischer Sicht besteht das Ziel, funktionale Zusammenhänge von Kultur und menschlichem Handeln zu analysieren. Triandis (1995) hat die von Hofstede (1980) eingeführte Typisierung von Kulturen nach ,individualistischen“ und ,kollektivistischen“ Kulturen aufgegriffen und theoretisch und empirisch differenziert weitergeführt. Damit sollen funktionale Beziehungen zwischen kulturellem Kontext und individuellem Handeln analy- 
siert werden. Triandis geht von einer Typologie aus, in der Kulturen gemäß ihrer Ausprägung von Individualismus und Kollektivismus auf einer horizontalen oder vertikalen Ebene sozialer Beziehungen beschrieben werden. Als horizontal gelten soziale Beziehungen, wenn sich die Kulturangehörigen als gleich verstehen. Als vertikal gelten Beziehungen, wenn sich Kulturangehörige als ungleich erleben und ihre Positionen auf einer sozialen Hierarchie anordnen. In individualistischen Kulturen sind die individuellen Zielsetzungen auf die eigene Person, und in kollektivistischen Kulturen sind sie auf die Gruppeninteressen bezogen; des weiteren sind in individualistischen Kulturen Selbstbeschreibungen auf das eigene Selbst und in kollektivistischen Kulturen auf die Gruppe bezogen. Dies sind nach Triandis (1995) die eigentlichen Attribute, nach denen sich Kulturen beschreiben und abgrenzen lassen.

Diese dichotome Beschreibung von Kulturtypen ist inzwischen heftig kritisiert worden, vor allem, weil keine Kultur eindeutig in den einen oder anderen Kulturtypus paßt, und weil die beiden Kategorien sich nicht unbedingt ausschließen müssen. Darüber hinaus stellt sich die Frage, ob solche Typisierungen für die Beschreibung von Kulturen in einer Welt mit zunehmend verflochtenen Kulturen eher besser oder weniger gut brauchbar sind (Trommsdorff 1998a). Dies hängt mit der Frage zusammen, ob Verflechtungen und Kulturkontakte die Ausprägung von Kulturbesonderheiten eher verstärken oder abschwächen. Wird eine Differenzierung von Kulturen im Zuge von Globalisierung obsolet? Erfolgt im Zuge weiterer individueller Verfügbarkeit über Information, weiterer Technologisierung und Modernisierung auch bisher traditioneller Kulturen eine Zunahme von horizontalen sozialen Beziehungen und Bevorzugung individueller Zielsetzungen und Selbstbeschreibungen, d.h., erfolgt weltweit eine Veränderung kollektivistischer in Richtung individualistischer Kulturen, also eine Dominanz einer spezifischen Kulturprägung?

Im folgenden werden zwei Thesen diskutièrt, die eine Typologisierung von Kulturen nach Triandis problematisieren:

a. Kulturen sind komplex und unterliegen ständigem Wandel und

b. kulturspezifische Einzelmerkmale entfalten ihre Wirkung aus dem Gesamtkontext.

Komplexität und Wandel von Kulturen

Der Typologisierung von Kulturen als „universalistisch“ versus ,individualistisch" widerspricht, dass Kulturen vielgestaltig und komplex sind, wie dies auch für soziale Institutionen und Interaktionen innerhalb von Kulturen gilt. Zudem sind Kulturen selten isoliert und daher keineswegs stabil, besonders wenn Begegnungen mit anderen Kulturen erfolgen. 
So ist Japan z.B. heute schwer nur als kollektivistische Kultur zu beschreiben. Zwar ist dort gemäß konfuzianischem Denksystem traditionell über Vertikalität sozialer Strukturen hinaus eine hohe Gruppenorientierung typisch (Mishima 1993). Gegenwärtig sind jedoch eher horizontale Strukturen mit vertikalen vermischt, und es sind Individualisierungstendenzen erkennbar (Trommsdorff, Suzuki \& Sasaki 1990).

Zudem sind Definitionsversuche, wie z.B. die Definition von Triandis, dass kollektivistische Kulturen der Eigengruppe einen höheren Wert geben als der Außengruppe, zu präzisieren. Heißt Eigengruppe die eigene Kernfamilie, die erweiterte Familie (Clan), die Gemeinde, der Staat, oder ein überregionaler Bereich wie der Kontinent? In Japan weiten sich Eigengruppenzugehörigkeiten im Lebenslauf aus. Die Zugehörigkeit zur Gruppe des Kindergartens und der Schule wird später erweitert um die Universität und die Firma. Die Bindungen an diese Gruppen und deren Mitglieder sind emotional und sozial bedeutsam und von normativen Verpflichtungen gestützt. Dabei können sich Zügehörigkeiten zu verschiedenen Gruppen überlappen. Relevant sind diese Zugehörigkeiten vor allem deswegen, weil sie die soziale Identität des Einzelnen festlegen. Während Grenzen zwischen Eigen- und Außen(Fremd-)gruppe in Japan sehr klar definiert werden, sind Abgrenzungen einzelner Gruppenmitglieder voneinander schwach ausgeprägt. Vielmehr kann man im Sinne von Markus \& Kitayama (1991) in Japan von „interdependenten“ Identitäten im Gegensatz zu ,independenten Identitäten“ einer individualistisch orientierten Kultur sprechen.

Allerdings erweist sich aufgrund beträchtlichen Wandels im Zuge der wirtschaftlichen Rezession, die wiederum teilweise durch Globalisierungsprozesse bedingt ist, dass die bisher behauptete relative Homogenität der japanischen Gesellschaft durch beträchtliche soziale Ungleichheit abgelöst wird (Trommsdorff 1996, 1998b). In weitaus heterogeneren Kulturbereichen - wie in Indien - sind verallgemeinernde Deutungen einer universalistischen Kultur kaum noch haltbar. Zudem ist hier der Einwand berechtigt, dass in der hinduistischen Kultur trotz strenger Kastenregeln in hohem Maße individualistische Werte gelten, insofern als das Individuum verantwortlich für sein Leben, sein Kharma angesehen wird; damit sind soziale Verantwortungen für die engeren oder weiteren sozialen Gruppen (Familie, Kaste) eher sekundär und diffus.

Weiter darf neben der internen Komplexität von Kulturen nicht übersehen werden, dass sich die Kulturen selbst wandeln, sei es aufgrund externer oder interner, z.B. wirtschaftlicher oder politischer Veränderungen. Auf der anderen Seite können externe und interne Wandlungen und damit verbundene Anforderungen zu einer Stabilisierung von Kulturen und traditionellen kulturellen Werten führen. Dies zeigt sich besonders deutlich in islamischen Kulturen, die ihre traditionellen Besonderheiten reaktivieren sowie auch in asiatischen Kultu- 
ren, die ihre traditionellen Werte für eine erfolgreiche wirtschaftliche Entwicklung instrumentalisiert und verstärkt haben, wie das in Japan oder Südkorea der Fall gewesen ist.

Darüber hinaus birgt die generalisierte Beschreibung von Kulturen auf dem Niveau aggregierter individueller Persönlichkeitsmerkmale und Einstellungen (z.B. das Kollektivismus mit geringerem Selbstwertgefühl und mit geringerer Zufriedenheit bzw. Glück zusammenhängt) (vgl. Triandis 1995: 108) die Gefahr von vereinfachten Übergeneralisierungen, oder sogar ideologischen Verzerrungen. Unsere eigenen Untersuchungen zu Sozialisationsbedingungen in Israel, Indonesien und Japan zeigen zum Beispiel, dass Erziehungsziele und -methoden von Müttern unterschiedliche Anteile von kollektivistischen oder individualistischen Merkmalen aufweisen und dass diese unterschiedlich mit Einstellungen der Kinder zusammenhängen. Innerhalb der jeweiligen Kulturen, die u.a. nach Stadt-Land- und regionalen Unterschieden zu klassifizieren sind, vermischen sich ,kollektivistische" und ,individualistische" Merkmale kulturspezifischer Werthaltungen (vgl. Trommsdorff 1995).

\section{3} Funktionale Bedeutung kultureller Einzelmerkmale

Einzelmerkmale für Individualismus und Kollektivismus haben für sich genommen keinen Aussagewert. Vielmehr isterforderlich, solche singulären Beschreibungsdimensionen in ihrer funktionalen Bedeutung eingebettet in den kulturellen Kontext zu erfassen. Dies ist auch für Fragestellungen relevant, wie sie zur Migration in diesem Band behandelt werden. Erst bei Einordnung spezifischer Sozialisationsmerkmale in den kulturellen Kontext lassen sich Prognosen für die Sozialisationswirksamkeit und die Entwicklung von Kindern und Jugendlichen machen.

So ist das in westlichen Kulturen wichtige Erziehungsziel „Selbständigkeit“ für sich genommen kein brauchbarer Indikator für individualistische Kulturen. Dieses Erziehungsziel wird auch von koreanischen, indonesischen und japanischen Müttern als sehr wichtig angesehen; allerdings hat es dort einen ganz anderen Stellenwert. Dieses Erziehungsziel wird dort nicht nur zu einem späteren Entwicklungszeitpunkt gefordert, es hat auch eine andere Funktion, insofern als es nicht primär der Entlastung der Eltern dienen soll, damit diese mehr „Freiraum" erhalten, sondern indem das Kind durch Erlernen von Selbständigkeit primär der Erfüllung von sozialen Rollen im sozialen Kontext nachkommen kann. Somit ist das Erziehungsziel „Selbständigkeit“" in kollektivistischen Kulturen in Asien, aber auch in Südamerika, eng verknüpft mit dem Erziehungsziel Gehorsam im Sinne der Pflichterfüllung (vgl. Friedlmeier 1995; Trommsdorff 1985, 1995). 
Daher beurteilen japanische Jugendliche, die primär Selbständigkeit und weniger Gehorsamkeitsanforderungen ihrer Eltern erlebt haben, ihre Eltern als weniger emotional zugewandt - ganz im Unterschied zu deutschen Jugendlichen (vgl. Trommsdorff 1985, 1995). Ähnliche Befunde zeigen sich übrigens auch bei koreanischen Jugendlichen in Südkorea im Vergleich zu koreanischen Jugendlichen, deren Eltern in die USA ausgewandert sind, und die sich den westlichen Erziehungsmodellen angepaßt haben (vgl. Rohner \& Pettengill 1985).

In Befragungen japanischer und deutscher Studierender zusammen mit Kashiwagi haben wir nachweisen können, dass in beiden Kulturen die gleichen Persönlichkeitsmerkmale, wie Egoismus, abgelehnt werden. Auf der anderen Seite bestehen erhebliche Kulturunterschiede. Bei Deutschen wird die Verbindung von Eigenständigkeit, Sensibilität und Durchsetzung, bei Japanern sozial integratives Verhalten und Hilfsbereitschaft bevorzugt (unveröffentlichte Daten).

Neben erheblichen Kulturunterschieden überlappen sich Werthaltungen individualistischer und kollektivistischer Kulturkontexte. $\mathrm{Zu}$ prüfen ist, in welchen Bereichen Unterschiede bestehen und welche Bedeutung diese haben. Eine weitere Frage ist, ob diese Unterschiede durch interkulturelle Verflechtungen und Globalisierungsprozesse in Zukunft verschwinden, z.B. weil ein Wertewandel kollektivistischer Kulturen in Richtung auf individualistische Werte erfolgt?

Abgesehen einmal davon, dass diese Frage zu vereinfachend typisiert und dabei die bereits erwähnten Differenzierungen von komplexen und im Wandel begriffenen Kulturen ignoriert, würde diese Frage unter der Perspektive der Modernisierungs- und Globalisierungsannahme insofern zunächst bejaht werden, als bisher Modernisierung als eine Verstärkung von Individualisierungstendenzen westlicher Prägung verstanden wurde. Dass Individualisierung ein wünschenswertes Ziel gesellschaftlicher Entwicklung bedeutet, wird jedoch heute von Kritikern der Postmoderne bezweifelt, die Probleme der Individualisierung, wie Partikularismus, Relativismus, Fragmentierung und Pluralismus (von Lebensstilen, Geschlecht, Alter, Kulturen, etc.), dem Verlust von sozialer Verantwortung, Anomie diskutieren. Der Zwang zu Entscheidungen zwischen vielfältigen Alternativen wird danach nicht als Vorteil, sondern als Belastung gesehen, und die Beliebigkeit von Werthaltungen wird als Aufforderung für willkürliches Handeln gedeutet. Individualisierung impliziert eine Relativierung kultureller Werte; und Akzeptanz von Multikulturalität impliziert schließTich eine rechtswirksame Übernahme verschiedenster kultureller Verhaltenssysteme, die grundsätzlich im Widerspruch zu bestehenden individuellen Rechten stehen können (wie z.B. die islamische Regelung der Beschneidung 
oder der Verheiratung der Tochter in einem westlich-demokratischen Rechtssystem).

Diese Folgen der Individualisierung haben Fragen nach allgemeinen verbindlichen Werten zugespitzt. In der Kommunitarismus-Debatte wird z.B. versucht, einen krassen Ellbogen-Individualismus in einen „kollektivistischen“ Kontext sozial-verantwortlichen Handelns zu transformieren. Ob und welche institutionellen und vor allem kulturellen Voraussetzungen für einen individuellen Entwicklungs- und Handlungsrahmen bei der Verknüpfung von kollektivistischen und individualistischen Werten im Sinne einer individuellen sozialen Verantwortung geschaffen werden können, und ob durch internationale Verflechtungen, Globalisierung und damit verbundene Kulturbegegnungen eine solche Verknüpfung kollektivistischer und individualistischer Werte erfolgen kann, ist eine empirische Frage. Im folgenden werden vier Thesen zu möglichen Folgen zunehmender internationaler Verflechtungen von Kulturen diskutiert.

\section{Vier Thesen zur Internationalisierung von Kultur}

Internationale Verflechtungen bedeuten immer Begegnungen von Kulturen. Forschungen zu Kulturbegegnungen, ihren Bedingungen, Ausprägungen und Folgen legen nahe, im Sinne von Berry (1990), die Frage nach Verknüpfungen von Merkmalen verschiedener Kulturen aus der Sicht der Bereitschaft und Kompetenz der jeweiligen Kulturangehörigen zu behandeln. Kulturangehörige unterscheiden sich in ihrer Bereitschaft, überhaupt Beziehungen zu anderen Kulturangehörigen einzugehen und in ihrer Bereitschaft, ihre eigene kulturelle Identität zu bewahren. Je nachdem sind die Folgen von Begegnungen zwischen Angehörigen verschiedener Kulturen (z.B. von Majoritäts- und Minoritätskulturen) sehr unterschiedlich: Sie können auf Integration, Assimilation, auf Separation oder Marginalisation der Minoritätskultur hinauslaufen, wozu es viele empirische Beispiele gibt. Das Modell von Berry ist zwar für Migrationsfolgen entwickelt worden. Hier wird vorgeschlagen, es auch für Kulturbegegnungen im internationalen Raum anzuwenden. Als Folgen von Kulturbegegnungen im international Raum lassen sich differenzieren:

1. Es besteht eine einheitliche, durch Globalisierung entstandene ,internationale" Kultur, bei der

(a) die verschiedenen früheren Minoritätskulturen von (den Werten) einer bestimmten Majoritätskultur (z.B. USA, China, Islam) dominiert werden (Einheitskultur mit Majorisierung);

(b) Majoritätskulturen, die Besonderheiten der Minderheitenkulturen und umgekehrt) absorbieren und alle Kulturen in gleicher Weise vertreten sind, wobei eine Assimilation der verschiedenen Kulturen erfolgt ist. 
Bei dieser Entwicklung sind die verschiedenen Werthaltungen in einer „Weltkultur“ integriert.

2. Verschiedene Kulturen sind voneinander abgegrenzt. Dabei

(a) kann es zu mehr oder weniger konfliktreichen Kulturbegegnungen kommen und es

(b) können einige Kulturen marginalisiert werden, wobei die verschiedenen Kulturen nebeneinander bestehen.

Welche Bedingungen führen zu der einen oder anderen Entwicklungsform? Betrachten wir dazu vier Thesen zu Folgen von Kulturbegegnungen.

Im Globalisierungsprozeß technologisch fundierter interkultureller Vernetzungen entwickelt sich eine "internationale Kultur “. Ist das eine realistische Vision? Werden dann auch der Kulturbegriff und Anstrengungen für die Definition von Kultur obsolet?

(a) Mit Globalisierung sind hier die durch Modernisierung und Technisierung (z.B. durch Motorisierung, Elektrifizierung, Elektronisierung, moderne Informationssysteme) entstandenen Prozesse weltweiter Verflechtungen von nationalen Wirtschaften und Gesellschaften gemeint. Globalisierung beruht auf einer weltweit geteilten technologischen Entwicklung mit entsprechenden Folgen für die ursprüngliche Kultur. Technologisierung bedeutet u.a., dass die Steinaxt von der Stahlaxt ersetzt wird, inzwischen auch auf Papua Neuguinea; dass Pferd, Esel, Ochse und Wasserbüffel durch Traktoren ersetzt werden, auch auf abgelegenen Inseln im Südpazifik; dass die beschwerlichen Märsche Wasserlasten tragender Frauen durch - wie in abgelegenen Dörfern auf den Philippinen erprobt - solarenergiebetriebene Wasserpumpen überflüssig werden; oder dass zeitraubende Nachrichtenübermittlung durch Buschtrommeln heute von Transistorradio, Fernsehen, Video, Telefon oder anderen modernen Informationssystemen ersetzt werden. Diese Technologisierung begünstigt den Prozeß internationaler Verflechtungen und - psychologisch gesehen - den Austausch von Informationen und die Wahrscheinlichkeit der Angleichung von Erwartungen hinsichtlich gleicher Lebensbedingungen und damit das Interesse, sich an den Globalisierungsprozessen zu beteiligen.

(b) Weltweite Verflechtungen einzelner Nationen werden auch durch moderne Institutionen begünstigt, die inzwischen weltweit mehr oder weniger ausgeprägt 
zum jeweiligen lokalen Kulturgut gehören, wie eine differenzierte Schrift, staatliche Organisationen (mit entsprechenden Sanktions- und Kontrollsystemen wie Polizei), eine nach westlichen, d.h. Rationalitätskriterien geprägte Verwaltung sowie ein nach modernen medizinischen Prinzipien aufgebautes Gesundheitssystem, oder ein nach westlichem Muster strukturiertes Erziehungswesen mit entsprechenden Curricula. Die Vernetzung der Märkte erfordert darüber hinaus auch eine politische Deregulierung nationaler Märkte, was in hochmodernen Gesellschaften wie in Japan bisher jedoch nur eingeschränkt der Fall ist.

(c) Wenn internationale Verflechtungen durch Kulturbesonderheiten der beteiligten Akteure gestört werden, z.B. weil Gewohnheiten im Denken und Verhalten Konflikte erzeugen, können Strategien für die Angleichung kultureller Besonderheiten eingebracht werden, indem z.B. für , joint ventures“ international zusammengesetzte Teams eingerichtet werden, oder interkulturelles Lernen auf der Grundlage dafür entwickelter Lehr-Lernprogramme in der schule und im Betrieb erfolgt.

\subsection{Folgen von internationalen Verflechtungen}

Wir haben oben bereits gesehen, dass eine klare Trennung von Voraussetzungen und Folgen der Globalisierung schwer möglich ist, weil es sich hier um Wechselwirkungsprozesse handelt. Dennoch sei die Frage gestellt, welche Folgen internationale Verflechtungen für Kulturen haben können.

(a) Die Übernahme moderner Techniken und Institutionen und die Teilnahme am Prozeß internationaler Verflechtungen bedeutet für traditionelle Kulturen, Besonderheiten ihrer bisherigen Kultur teilweise oder ganz aufzugeben, wobei nicht ohne weiteres die Voraussetzungen bestehen, die neuen Handlungsoptionen erfolgreich aufzunehmen, so dass es zu dysfunktionalen Entwicklungen kommt. Ein Beispiel dafür sind die sozialen Probleme auf Papua Neuguinea, wo die Steinzeitkultur in kürzester Zeit durch moderne Technologien abgelöst wurde.

(b) Weltweite Verflechtungen von Märkten und Informationssystemen erweitern einerseits Handlungsoptionen wie Konsumangebote; andererseits bewirken sie auch Vereinheitlichungstendenzen, die nationale und kulturelle Grenzen überwinden. Vereinheitlichtes Konsum- und Freizeitverhalten wird Teil einer „McDonaldization of society" (Ritzer 1993). Coca Cola und Mc Donald's sind in allen Teilen der Welt zu finden, nicht nur in der Werbung, auch in Verhaltenspräferenzen. Das gleiche gilt für die Disney-Land-Kultur mit ihren Disney- 
Land-Zentren und -produkten von Kalifornien über Paris nach Tokio und Manila, oder für Rock und Techno, für Adidas und Tennisstars. Die Einheitskultur von Disneyland und Coca Cola wird in alle Teile der Welt durch Fast-FoodJournalismus in Medien vermittelt: Durch Fernsehen - und wenn es nur ein Gerät ist, das beim Dorfältesten oder auf den für die Beratung der ältesten Männer des Dorfes vorgesehenen, nach allen Seiten offenen Holzhauses eines abgelegenen thailändischen oder indonesischen Dorfes steht.

Das Bemühen um Teilhabe an der Einheitskultur geht ein in die Identitätsbildung, die über die Zugehörigkeit zu der bisherigen Kultur hinausgeht (Friedman 1994). Kinder werden in vielen Teilen der Welt frühzeitig zu begeisterten Konsumenten dieser Einheitskultur und übernehmen die entsprechenden vom Markt vorgegebenen Zugehörigkeitssymbole. Jugendliche partizipieren international in den verschiedensten Bereichen von Freizeit und Konsum an den schnell wechselnden Moden der Einheitskultur.

Dabei geht es auch um das richtige ,timing“ der Partizipation. Wenn man dazugehören will, muß man den neuen Trend rechtzeitig erkennen. Dass Kulturfiguren Trends definieren, ist nicht ohne den jeweiligen Zeitgeist - d.h. auch die Bedürfnislage der Konsumenten - möglich. Die Vermarktung erfolgt dort, wo auch entsprechende ökonomisch abgesicherte Konsumbereitschaft besteht. Konsumenten verleihen den Trendsettern Meinungsführungsmacht, ständig und schnell wechselnde Verbindungen zwischen Angeboten, Meinungen und Verhalten herzustellen. Diese wechselnden Erscheinungen einer Weltkultur von Konsum und Freizeit werden durch technologisch hoch entwickelte Informationsnetze und internationales Marketing global vermittelt (Kornadt 1986).

(c) Internationale Kultur äußert sich danach in weltweit relevanten Symbolwelten, wie sie in Kleidung, Eßkultur, Musik zum Ausdruck kommen und weltweit übergreifende Gemeinsamkeiten von Konsumstilen (Coca-Cola und Mc Donald 's) und Verhaltensweisen westlichen Musters aufweisen. Welche Werte mit dieser globalisierten Konsum- und Freizeitkultur verbunden sind, ist eine empirisch zu klärende Frage. Es erscheint bisher, dass damit westliche Werte von Freiheit, Unabhängigkeit, Fortschritt, Erfolg und Macht vermittelt werden, die in hohem Maße mit der amerikanischen Kultur assoziiert sind.

Dass auch andere westliche Werte im Globalisierungsprozeß als universell gültig vorgebracht werden, ist spätestens seit der Diskussion um Menschenrechte bekannt. Ähnlich gilt dies teilweise auch für Werte der Umwelterhaltung, der Demokratie und der Deregulierung, die vor allem auf Seiten westlicher Gesellschaften als gültig bzw. überlegen verbreitet werden. Mit der Veränderung der weltpolitischen Kräfteverhältnisse, aber auch seit dem wirtschaftlichen Einbruch in Ostasien und Südostasien scheinen sich diese westlichen 
Werte als „erfolgreich“ bewährt zu haben. Der Zusammenbruch der Sowjetunion und des Kommunismus haben Werte der freien Marktwirtschaft, Demokratie und Freiheit als Modell und Grundlage einer umfassenden Weltkultur begünstigt. Bei gegenwärtiger Bedeutung von wirtschaftlichem Wachstum müßte dieses Modell für eine internationale Kultur die größten Chancen haben, weil es sich bisher als erfolgreich erwiesen hat, auch wenn dabei die Frage nach hinreichenden und notwendigen Voraussetzungen für den Erfolg nicht beachtet wird.

\subsection{Zusammenfassung}

Diese Phänomene sind Grund für die erste These, dass ein Trend zu einer globalen Kultur besteht, in der die zwischenmenschliche und interkulturelle Kommunikation über die Kontinente hinweg vereinfacht und in einer grenzüberschreitenden internationalen Kultur eingebunden sind. Diese These lautet daher also, wenn in einer durch Globalisierung bzw. in einer durch wirtschaftliche Interdependenz und moderne Kommunikationssysteme vernetzten Welt allmählich politische und kulturelle Grenzen an Bedeutung verlieren, ist zu erwarten, dass sich eine Einheitskultur international entwickeln wird.

2.

Regionalisierungsthese

Der Globalisierungsthese soll im folgenden eine zweite These - die Regionalisierungsthese - gegenübergestellt werden. Es erfolgt eine zunehmende Ethnisierung bzw. eine Verstärkung religiöser Gruppierungen und eine Regionalisierung von Kultur. Dazu lassen sich viele Beispiele nennen.

(a) Die relativ stabile bipolare Machtaufteilung der Nachkriegszeit ist heute einer multipolaren Machtverteilung gewichen, in der neue Blöcke wirtschaftlicher Machtzentren entstehen, die jeweils durch Zusammenschlüsse verschiedener Regionen mit kulturellen Gemeinsamkeiten gekennzeichnet sind: Der abendländische-europäische Kulturraum mit Europa und den USA oder der asiatisch-pazifische Raum mit Japan als wirtschaftlichem Führer der fünf Tiger. Diese multipolare politische Entwicklung nach dem Zusammenbruch des Ostblocks ist nicht nur von wirtschaftlichem und politischem, sondern vor allem auch von kulturellem Interesse bestimmt, und zwar hinsichtlich der Bedeutung kultureller Identität.

Nach dem Zerfall der UdSSR sind massive Bestrebungen der Unabhängigkeit ethnischer Gruppen entstanden, die zur Gründung neuer Republiken und häufig zu blutigen Auseinandersetzungen, verbunden mit ,ethnischen Säuberungen“, wie im ehemaligen Jugoslawien geführt haben. Auch in Westeuropa 
verstärken sich solche regionalen Bewegungen mit einer grenzüberschreitenden Revitalisierung des Keltisch-Gälischen (Schotten, Iren, Walliser, Bretonen), oder Konflikten zwischen Schottland und England. Die norditalienische Liga, Basken und Korsen fordern verstärkt ihre Unabhängigkeit und kämpfen dafür z.T. mit terroristischen Mitteln. Auch außereuropäische Beispiele belegen die Zunahme regionaler Bewegungen. In Ruanda und Zaire sind ethnische Konflikte Grund für kriegerische Auseinandersetzungen, wirtschaftlicher Not und Massenelend.

Allerdings müssen Regionalisierungstendenzen nicht immer zu konflikthaften Auseinandersetzungen führen. Die Förderung ethnischer Besonderheiten kann, wie das Beispiel Indonesien zeigt, auch ein politisches Ziel für die Entwicklung einer nationalen Identität sein. Dort werden die vielen ethnischen Gruppen in ihren kulturellen Besonderheiten geschützt und gestärkt, um als eigene Kulturgruppen politisch integriert zu werden. „Einheit durch Vielfalt" (Pancalisea) lautet das offizielle Motto. Allerdings zeigt sich die Brüchigkeit solcher Ideologien in Krisenzeiten. In der gegenwärtigen Wirtschaftskrise hat es die chinesische Minderheit in Indonesien aus verschiedenen Gründen schwer. Chinesen werden (und fühlen sich) bedroht; sie gelten als Sündenbock für die anstehenden Probleme. Die Regionalisierung und Abgrenzung von Kulturen (im Gegensatz zu einer Internationalisierung) kann also eine Herausforderung an die Kulturen und gesellschaftlichen Institutionen bedeuten, wenn im Prozeß der Aktivierung regional gebundener Kulturen neue Konfliktbereiche entstehen.

(b) Regionale Kulturbesonderheiten werden trotz Globalisierung von Wirtschaft, Technologie und Kommunikation erhalten. Eigene Kulturbesonderheiten haben in Japan sowie auch für Schwellenländer wie Thailand und Indonesien Priorität. Ein Kentucky Fried Chicken Logo in Japan ist insofern japanisiert, wenn dies im Stil den üblicherweise in den Schaufenstern von Restaurants ausgestellten Imitationen von Speisekarteninhalten nahe kommt. Es gilt auch heute nicht als Normverletzung, wenn eine Geisha im Kimono zu Mc Donald 's essen geht. Japanische Punks, die sich angeblich gegen den übermächtigen Druck der Harmonie und Anpassung fordernden eigenen Gesellschaft auflehnen, treffen sich am arbeitsfreien Sonntag (sonst gehen sie geregelten Arbeiten nach) in einem Tokioer Stadtviertel in Harajuku und geben sich in teurem Outfit ein Stelldichein, das von globalen Märkten bzw. internationalen Medien und Modefirmen inszeniert zu sein scheint, das aber gleichzeitig die kulturelle Tradition einer verschwenderisch eleganten Verpackung überflüssiger Kleinigkeiten imitiert. Daneben nimmt sich z.B. eine „Love-Parade“ in Berlin mit ihrem postmodernen Hedonismus-Konzept schrill und unartikuliert aus. 
Es gibt viele Beispiele für partielle Vermischungen von fremden und eigenen Kulturelementen. Die japanische Kultur ist dafür berühmt, ausländische Einflüsse aufzunehmen und zu amalgamieren, ohne dabei die eigene kulturelle Identität zu verlieren. Das gilt für Technik, Wirtschaft, Kunst und Politik. Die geschickte Selektion von technischen Entwicklungen aus anderen Kulturen und deren Weiterentwicklung im eigenen Land hat in Japan zu großen Erfolgen geführt. Ein Beispiel ist, dass in Japan die deutsche Erfindung des Wankelmotors aufgegriffen und erfolgreich weiterentwickelt wurde. Ähnliches gilt für die Übernahme von Teilen des deutschen Rechtssystems oder der deutschen Medizin in der Meiji-Zeit.

Solche Beispiele kultureller Mischungen zeigen, dass eine gewisse Übernahme fremder Kulturelemente in die eigene Kultur erfolgreich sein kann. Dies kann jedoch eine nur scheinbare Anpassung der eigenen an die andere Kultur bedeuten. Bestimmte Kulturbesonderheiten können eine Teil-Integration erlauben, ohne dass die eigenen kulturellen Besonderheiten aufgegeben werden. So können neue Misch- und Subkulturen entstehen, die selektiv z.B. westliche Individualisierungssymbole ubernehmen und so durch den eigenen Kulturkontext filtern, so dass sie konfliktlos in die eigene Kultur eingebunden werden können. Daher sind (z.B. durch wirtschaftliche Interessen vermittelte) Vereinheitlichungstendenzen in Verhaltensstilen und Identitäten keineswegs bereits Indikatoren einer Weltkultur.

Im übrigen ist bekannt, dass sich z.B. eine schlichte Übernahme einzelner Prinzipien erfolgreicher japanischer Betriebe (wie der Personalführung und des Management) in deutsche oder amerikanische Betriebe selten bewährt hat. Aus dem kulturellen Zusammenhang gerissen funktionieren solche Einzelmaßnahmen nicht. Außerdem scheint es kulturspezifisch unterschiedliche Bereitschaften zu geben, von anderen Kulturen zu lernen und Merkmale anderer Kulturen aufzunehmen.

Zusammenfassend ist zu sagen, dass die Bedeutungsmacht der Eigenkultur nicht nur zur Folge haben kann, dass fremde Kulturelemente abgestoßen oder integriert werden. Sie kann auch zur Folge haben, dass trotz politisch, wirtschaftlich und technologisch weiterentwickelter Globalisierung lokale ,indigineous" Kulturen bestehen bleiben, ja teilweise sogar gestärkt werden, und ihre spezifische Bedeutungsmacht nicht in einer ,internationalen“ Kultur aufgelöst wird.

(c) Warum ist neben einer Globalisierung gegenwärtig eine starke Regionalisierung mit zunehmender Bedeutung von ethnisch und regional gebundenen Kulturen zu beobachten? Zum einen ist diese Regionalisierung wohl eine Antwort auf Schwierigkeiten lokaler und regionaler Wirtschaftseinheiten, die durch den erheblich gewachsenen Konkurrenzdruck im Zuge der Globalisierung ent- 
stehen. Zum anderen ist insbesondere die kulturelle Regionalisierung wohl eine Antwort auf bestimmte, mit den eigenen Werten nicht zu vereinbarende Handlungsweisen und -regeln (z.B. im wirtschaftlichen oder sozialen Verhalten) sowie eine Antwort auf unüberschaubare, unkontrollierbar erscheinende und damit identitätsbedrohende Handlungsprozesse in Wirtschaft, Politik und Gesellschaft.

Zusammenfassend ist eine Aktivierung lokaler, regionaler und ethnischer Kulturen zu beobachten, was im Kontrast zu Internationalisierungs- und Globalisierungstendenzen steht.

3.

Auflösung regionaler Kulturen

Sind aber nicht gleichzeitig auch Auflösungen lokaler bzw. regionaler Kulturen zu beobachten? Damit sind wir bei der nächsten These: Es erfolgt eine zunehmende Auflösung lokaler Kulturen.

(a) Nicht nur Anthropologen und Missionare, auch Marketingexperten und Entwicklungshelfer haben inzwischen alle Teile dieser Welt „entdeckt“" und Steinzeitkulturen aussterben lassen. Daher lassen sich heute kaum noch ,ursprüngliche" unberührte Kulturen finden. Heute sind alle Kulturen auf irgendeine Weise mit dem westlichen technologischen Fortschritt in Berührung gekommen, haben sich mit diesen westlichen Kulturprodukten auseinandergesetzt, diese teilweise aufgenommen und dadurch ihre eigenen Existenzgrundlagen verloren.

Einige Kulturen sind dadurch erheblich dezimiert, wie die Iban auf Borneo, oder die Kalahari-Bushmen in Afrika. Andere Kulturen sind zugrunde gegangen, wie die Aborigines in Australien; oder sie löschen sich gegenseitig aus, wie die Tutsi und Hutu. Wieder andere Kulturen vollziehen tiefgreifende Wandlungsprozesse, von denen noch nicht klar ist, was von der ursprünglichen Kultur bestehen bleibt, und wie weit sie fremde Einflüsse assimiliert, wie z.B. traditionelle Kulturen im Südpazifik, die inzwischen auch für den JedermannTourismus erschlossen sind wie auf Bali oder den Trobriand-Inseln.

Mit der Modernisierung und Industrialisierung verlieren sogenannte „indigineous“ Kulturen ihr traditionelles Kulturgut, d.h. z.B., dass sich ihre Sprache verändert. Die Tradition mündlich überlieferter Regeln und Weisheiten weicht der Verschriftung von Wissen, was einer inzwischen (teilweise) alphabetisierten Bevölkerung zur Verfügung steht. Bisheriges Experten- oder gar Geheimwissen mit den entsprechenden Autoritäts- und Sozialstrukturen verschwindet. Z.B. hat traditionell der Häuptling oder Medizinmann die Rechtsprechung, die Verteilung von knappen Nahrungsressourcen bis zur nächsten 
Ernte, die wirtschaftliche Versorgung und soziale Integration seines Dorfes sicher gestellt und traditionell die Verbindung mit den göttlichen Kräften und die Verantwortung für magischen Zauber übernommen. Jahrtausendelang funktionierende Kenntnisse über Techniken der Geburtenkontrolle, Heilungstechniken, Methoden beim Jagen und Fischen (z.B. Gebrauch von Blasrohr und Harpune) oder für den Anbau landwirtschaftlicher Produkte - diese Kenntnisse verschwinden, wie bei den Eskimos oder den Dajak auf Borneo.

Die soziale Integration durch die gemeinsame Ahnenkultur, die Weitergabe gemeinsamer Werte an die nächste Generation, der Stolz auf die eigene Gruppe und Gruppensolidarität - diese kulturellen Ressourcen gehen verloren. Verbindliche Regeln und soziale Verpflichtungen werden eingetauscht gegen Autonomie und Freiheit; sowie gegen Anomie und Verlust an sozialen Beziehungen. Der Motor für diesen Kulturwandel, für den Übergang in die „Modernisierung", wie wir dies in vielen Teilen der Welt beobachten kọnnnen, sind die Hoffnung auf materiellen Wohlstand, auf Reichtum und ein bequemes Leben. Häufig erfolgte dieser Wandel nicht freiwillig, sondern wurde durch Kolonialisierung, Missionstätigkeiten, Tourismus oder andere politische und ökonomische Eingriffe von außen herbeigeführt.

(b) Auch innerhalb von Gesellschaften können ,indigineous“ Kulturgruppen verschwinden. Was bedeutet es für eine Gesellschaft, wenn bisher funktionierende und integrativ wirkende Teilgruppen mit eigenständigen Kulturbesonderheiten zum Anachronismus werden? Ein Beispiel sind zunftähnliche Berufsgruppen, in denen Verhalten durch Rituale, Symbole, und kulturelle Identität als Mitglied dieser Zunft organisiert sind. Handwerker haben traditionell eine besondere Sozialisation unter Betonung von Werten der Sorgfalt und Regeleinhaltung erfahren. Bauern wurden traditionell unter Betonung von Werten der langfristigen Planung, der Akzeptanz von Naturphänomenen und Frustrationstoleranz, der Genügsamkeit, Geduld und Ausdauer sozialisiert.

Wenn solche Gruppen, wie z.B. der deutsche Bergbau oder die Landwirtschaft durch Steuergelder subventioniert werden müssen, um den Funktionsverlust zu kompensieren, kann dies eine Identitätsbedrohung für die Mitglieder dieser Gruppen nicht verhindern; für die Gesamtgesellschaft bedeutet das Verschwinden einer solchen Kulturgruppe ein Verlust an Werten wie Solidarität, Genügsamkeit und Bescheidenheit.

Die Handwerkerzünfte in Europa und die Reisbauernkultur in Japan sind Beispiele für wirtschaftliche produktive Gruppen, die kulturelle Werte tradiert und damit lange Zeit wichtige Funktionen für die soziale Integration der Gesellschaft übernommen haben. Wie anders können solche kulturellen Ressourcen tradiert und wirksam werden, wenn nicht entsprechende Kulturgruppen als ihre Träger fungieren? Bei Schrumpfen z.B. der deutschen oder japanischen Agrar- 
wirtschaft auf unter $10 \%$ der Bevölkerung ist eine solche Funktion schwer realisierbar.

Sind solche kulturellen Teilgruppen heute anachronistisch, und sind die von ihr vermittelten und vertretenen Werte dysfunktional für die Bewältigung sozioökonomischer Umbrüche und Postmodernisierungstendenzen?

Das Verschwinden wirtschaftlicher Teilkulturen kann erhebliche Folgen für einen Wertewandel der Gesellschaft haben. In Japan beruht die schnelle und erfolgreiche Industrialisierung auf Werthaltungen, wie sie durch die traditionelle agrarwirtschaftliche Kultur des Naß-Reisanbaus vermittelt wurden. Dazu gehören u.a. eine differenzierte Arbeitsteilung, hohe Normorientierung und Gruppensolidarität, um die Bewässerung zu regeln. Die in Japan traditionell vermittelten Werthaltungen, Sozialisationsziele, Kulturtechniken und Verhaltensweisen, die den gegebenen klimatischen und ökologischen Bedingungen des Landes angepaßt sind, haben offensichtlich dazu beigetragen, den Übergang in eine erfolgreiche Modernisierung zu vermitteln. Voraussetzungen dafür bestehen in psychologischen und sozialen Bereitschaften, den Anforderungen der modernen Industriegesellschaft und damit verbundenen gesellschaftlichen Umbrüchen mit einem hohen Maß an Frustrationstoleranz, Leistungsmotivation und Gruppensolidarität zu begegnen (Mishima 1993; Suzuki 1993). Diese Ressourcen lassen sich als japanische Besonderheiten sehen, die nicht nur zu dem beeindruckenden Wirtschaftserfolg Japans beigetragen haben, sondern die auch das Potential aufweisen, Japan aus seiner Wirtschaftsrezession und seinen gegenwärtigen gesellschaftspolitischen Krisen herauszuführen (vgl. Trommsdorff 1998b).

(c) Das führt zu der Frage, welche Folgen das Verschwinden von Kulturgruppen für die Anpassungsfähigkeit einer Gesellschaft bzw, ihrer Mitglieder an Anforderungen sozioökonomischen Wandels hat. Wenn man die Diskussion um den Kommunitarismus und die Debatte um das Wiederaufleben traditioneller Werte der Leistung, Loyalität, sozialen Verantwortung und Wertebindung verfolgt (vgl. Etzioni 1994), so müßte man eher zu dem Schluß kommen, dass diese „anachronistischen“ Teilkulturen Werthaltungen vertreten und vermitteln, für die allgemein heute ein hoher Bedarf besteht. Lassen sich sozio-ökonomische Anachronisten als Träger zukunftsweisender kultureller Werte sehen, die durch Globalisierungsprozesse an Bedeutung gewonnen haben? Wenn jedoch solche zukunftsrelevanten Werte wirklich gebraucht werden, wie lassen sich diese heute vermitteln? Welche kulturellen Teilgruppen können (mit in dieser Hinsicht funktional äquivalenten Funktion) für die Sozialisation solcher Werte Anfrage kommen oder gehen diese Werte und die Möglichkeit für ihre Vermittlung mit dem Verschwinden der sie traditionell tragenden Kulturgruppen zum Nachteil der Gesellschaft verloren? 
(d) Anders stellt sich die Frage für den - durch Modernisierungsprozesse erfolgenden - Funktionsverlust von traditionellen Eliten. Aufgrund von Charisma und Expertenwissen sind Eliten für die Lösung individueller, sozialer und wirtschaftlicher Probleme zuständig gewesen. Durch Aneignung rational-technokratischen Expertenwissens können Eliten einen Teil nunmehr anachronistischen Wissens kompensieren. Kulturträgergruppen von Eliten, die aus Gründen der technischen und ökonomischen Entwicklung aussterben, waren weniger direkt an der wirtschaftlichen Produktivität beteiligt; vielmehr haben sie Kulturwissen elitär verwaltet und als Geheimwissen z.B. zur Stabilisierung der eigenen Macht und der Globalisierung sozialer Beziehungen in der Gemeinschaft benutzt - z.B. Heilige in Indien, Schamanen in Korea, Medizinmänner bei den Hutu. Traditionelle sozial-integrative Eliten werden in modernen Industrienationen ersetzt durch Experten, die über objektivierbares, rational begründetes, und grundsätzlich allgemein verfügbares problemlöserelevantes Wissen verfügen, oder durch populäre Medien artikulieren. Können diese modernen Experten die Funktion traditioneller Eliten übernehmen?

(e) Zusammenfassend lautet die dritte These, dass mit einer Globalisierung ein Verlust traditioneller Kulturen mit ihren grundlegenden Werten und Überzeugungen einhergeht. Wir bewegen uns in Richtung auf eine internationale Kultur und geben traditionelle kulturelle Werte und traditionelle Bindungen auf.

4.

Revitalisierung von Kulturen

Dieser These halte ich eine vierte These entgegen: Eigenständige Kulturen gewinnen an Bedeutung.

In den verschiedenen Gesellschaften, die von der Globalisierung betroffen sind, bestehen historisch vermittelte Kulturbesonderheiten und damit verbundene Werthaltungen und Kompetenzen. Dies sind Ausgangsbedingungen, durch die die neuen Anforderungen überhaupt erst einmal subjektiv wahrgenommen, aufgenommen aber vielleicht auch abgewehrt werden. Daher ist anzunehmen, dass Menschen mit den marktvermittelten Angeboten (z.B. Konsum) zur Partizipation an einer Weltkultur nicht in gleicher Weise umgehen. Je nachdem, wie Angebote im Kontext der jeweiligen Kultur eingebettet sind und ob diese Angebote tatsächlich vom einzelnen Konsumenten als identitätsstützend wahrgenommen werden, wird die Zugehörigkeit zur eigenen Kultur gestärkt.

(a) In vielen Gesellschaften bestehen gegenwärtig keine oder geringe ökonomischen Voraussetzungen für den Konsum bzw. die Teilhabe an der Mc Donald's Kultur. Osteuropäische Staaten haben z.B. nach der Auflösung des Ostblocks eher die Priorität, eine Wirtschaftsstabilisierung zu erreichen und ihre 
nationale Identität abzusichern. In anderen Gesellschaften besteht massiver Widerstand gegen westlichen Fortschrittsglauben und westliches Konsumdenken. So verstehen die durch den islamischen Fundamentalismus repräsentierten Gegner der westlich-arnerikanischen Individualisierungs- und Fortschrittsidee unter Globalisierung und internationaler Kultur eben nicht die Mc Donaldisierung von Gesellschaften, sondern die Verbreitung des islamischen Fundamentalismus. Dies ist gegenwärtig im Erstarken des Islam zu beobachten, ausgehend vom Iran in den arabischen Ländern, in großen Teilen Afrikas und der ehemaligen Sowjetunion, sowie inzwischen in Malaysia und Indonesien und neuerdings auch an der Grenze zu Tachekistan (mit Konflikten zwischen Moslems und Chinesen).

Während einige Gesellschaften also ökonomisch und sozial gar nicht in der Lage sind, an der Globalisierungsidee westlicher Prägung zu partizipieren, wehren sich andere Gesellschaften gegen diese Idee unter Verstärkung der eigenen Identität, oder es kommen beide Faktoren zusammen.

(b) In verschiedenen Kulturbereichen in Asien, in Indien oder in den arabischen Ländern beobachten wir eine deutliche Orientierung auf eigene kulturelle Traditionen bei gleichzeitiger Ablehnung einer Verwestlichung kultureller Werte, d.h. eine Ablehnung individueller Autonomie und hedonistischer Werte westlicher Prägung. Dies ist z.B. in fundamentalistischen Tendenzen des Hinduismus in Indien zu beobachten.

In Japan z.B. hat man erkannt, dass die traditionellen kulturellen Werte, die man als Grundlage des enormen wirtschaftlichen Erfolges ja der wirtschaftlichen Überlegenheit ansieht, inzwischen von westlichen Industrienationen in den USA und Europa imitiert werden - und das, nachdem Japan als die Kultur gegolten hat, die in hohem Maße westliches know-how und Können übernommen hat. Mit dem "nihonjiron“ besteht in Japan eine starke Revitalisierung eigener traditioneller, insbesondere partikularistischer Werte, diese widersprechen teilweise den mit der Globalisierung verbundenen universalistischen Werten (Trommsdorff 1996).

Mit einer Dichotomisierung von Universalismus und Partikularismus lassen sich jedoch die gegenwärtigen Tendenzen wohl kaum angemessen beschreiben. Vielmehr können in manchen Kulturen sowohl partikularistische wie universalistische Elemente gut verbunden werden, in anderen Kulturen schließt sich diese aus. So wird in Ostasien gegenwärtig beides gefördert - einerseits eine Hinwendung zu regionalen asiatischen Werten, insbesondere zum Konfuzianismus („Confucian Rennaissance“) (Heberer 1997) und andererseits eine Internationalisierung. Inwieweit der Konfuzianismus, mit dem Werte der Gruppenorientierung, Seniorität, Loyalität, Disziplin, Bescheidenheit und Pflichtorientierung verbunden sind, tatsächlich den asiatischen „Geist des Kapitalismus“ fördert, 
ist eine Frage an die kulturpsychologische Forschung. Mit der Rückkehr zu den eigentlich asiatischen Werten des Konfuzianismus wird im asiatischen Raum gegenwärtig die Lösung bestehender Probleme gesehen. In den eigenen ,indigenous" kulturellen Werten besteht für viele asiatische Intellektuelle eine Energiequelle, die es zu verstärken gilt, und zwar in Abgrenzung zu anderen, insbesondere zu westlichen Kulturen und im Sinne der Stärkung der eigenen Identität.

(c) Folgen von Globalisierungsprozessen könnten einerseits eine Internationalisierung von Kultur hervorbringen, wenn diachronisch kulturelle Entwicklungsverläufe und synchronisch unterschiedliche regionale Kulturformen zusammenwirken. Globalisierungsmodellen westlichen wirtschaftlichen Fortschritts und internationaler Kulturen stehen andererseits jedoch deutlich Gegenmodelle im Sinne des islamischen Fundamentalismus gegenüber. Die Folgen wären Konflikte zwischen Kulturen. Ein verstärkter Fundamentalismus im Sinne der Revitalisierung traditioneller kultureller Werte hat bereits jetzt in einigen Regionen zu massiven sozio-politischen und ökonomischen Umbrüchen geführt, wie z.B. in Afghanistan und Algerien. Eine Fanatisierung des Fundamentalismus und dem damit verbundenen Identitäts- und Abgrenzungswahn wird ein kulturübergreifendes multikulturelles Zusammenleben verhindern. Damit ist jedoch nicht gesagt, dass ein „Kampf der Kulturen“ (Huntington 1996) bevorsteht. Für das Modell der „clashes of cultures“ gilt eine ähnliche Kritik wie für die Individualismus-Kollektivismus-Typologie, die Kulturen als homogene, in sich und gegeneinander abgeschlossene Entitäten behandelt und ignoriert, dass sich normalerweise moderne Gesellschaften vielfältig ausdifferenzieren und dass zumindest Überlappungen von Kulturen zu erwarten sind.

Konsequenz der bisherigen Analysen ist die Feststellung, dass bei gegenwärtiger Ausgangslage eine Weltkultur in weiter Ferne steht.

\section{III . Psychologische Bedeutung von Kultur}

Nach der Diskussion dieser Thesen auf der Grundlage von Phänomenbeschreibungen und einiger psychologischer Implikationen stellt sich nun abschließend die Frage, ob die viel beschworene Globalisierung nur ein Schlagwort ist, um auf der Ebene technologisch-wirtschaftlicher Prozesse internationale Vernetzungen zu beschreiben (vgl. Friedrichs 1997), oder ob bestimmte kulturelle Veränderungen damit verbunden sind. Psychologische Prozesse, die mit der Globalisierung einhergehen, wie z.B. eine Rationalitätszunahme bei gleichzeitiger Entmythologisierung dürften erhebliche Verluste an emotional fundierten eigenen kulturellen Traditionen, eine Verflechtung und Vereinheitlichung von kulturellen Identitäten bei gleichzeitiger Verunsicherung und Identitätsbedro- 
hung bedeuten. Eine internationalisierte Kultur im Sinne einer auf Rationalität gegründeten Globalisierungsidee, die übrigens auch den Kulturbegriff obsolet macht, würde grundlegenden menschlichen Bedürfnissen nach Sicherheit, Geborgenheit und Zugehörigkeit, dem Bedürfnis nach metaphysischer Sinngebung, sei es in einer Religion, in einer Gemeinschaft oder in einer sozial und lokal verankerten Kulturgruppe widersprechen. Dies wäre Zündstoff und Dynamik für Konflikte und für Prozesse, bei denen Abgrenzungen von anderen Kulturen einhergeht mit Akzentuierungen der eigenen Kultur und wohlmöglich einer fundamentalistischen Orientierung.

1 .

Grundlagen der Beziehung von Person und Kultur

Die letzte These lautet daher: Menschen brauchen Kultur und wollen überschaubare Kulturbindungen.

Dieses angenommene Bedürfnis nach Kulturbindung sehe ich begründet in dem universellen Bedürfnis nach Zugehörigkeit und Geborgenheit in einer kleinen und überschaubaren Gruppe (z.B. Horde, Großfamilie, Produktionsgemeinschaft), einer Gruppe also, in der gegenseitiges Vertrauen und gegenseitige soziale Verantwortung eine sichere Lebensbasis bieten. Mit dem Verschwinden von verbindlichen Werthaltungen und übergreifenden Sinndeutungen und Geborgenheiten verschwinden nicht universelle Bedürfnisse nach identitätsstiftender Einbettung und Bindung. Es stellt sich daher die Frage nach den Grundlagen der individuellen und kulturellen Identität und ob eine Identität, die nicht primär auf kulturellen Werten, sondern auf einer Staatsverfassung oder einer Wirtschaftsgemeinschaft beruht, im psychologischen Sinne identitätswirksam sein kann. Hier gehe ich davon aus, dass dem Bedürfnis nach eigener Kultur ein Bedürfnis zugrunde liegt nach

- Erklärung und Verstehen sowie Sinngebung von Selbst- und Umwelterfahrungen;

- Handlungsorientierung und damit verbundenen handlungsrelevanten motivierenden Zielsetzungen sowie

- Geborgenheit und Zugehörigkeit, und damit nach Identität.

Das ist eine weitreichende Annahme, die jedoch entwicklungspsychologisch gut begründet ist.

Spätestens seit Piaget (1992) wissen wir, dass ein universelles Bedürfnis nach Verstehen und Kontrolle besteht und der Entwicklung des Menschen zugrunde liegt. Zunächst werden physische Gegebenheiten zu erklären versucht; später 
entwickeln sich weitreichende Deutungsmuster und kognitive Schemata, die schließlich auch transzendentale Deutungen umfassen. Dabei werden nicht nur rationale Erklärungen bevorzugt, vielmehr sind magische Erklärungen vielfach wirksamer. Solche Erklärungsversuche liegen einem universellen Bedürfnis nach Sicherheit und Kontrolle zugrunde.

Aus kulturpsychologischer Sicht erweist sich die emotionale Verankerung von Deutungen, wie sie durch traditionelle Überzeugungen und religiöse Systeme vermittelt wird, als besonders wirksam. Die durch Säkularisierungsprozesse verloren gegangenen emotionalen Grundlagen für Sinngebung und Deutung werden gegenwärtig vielfach durch Rückfall in primitive Denkmuster, reaktionäre Tendenzen und durch Hinwendung zu Sekten u.a. kompensiert. Je nach kulturellen Werthaltungen werden unterschiedliche Deutungsmuster favorisiert, die verschiedene Arten von Kontrolle und Handlungsorientierung vermitteln (vgl. Trommsdorff 1998a).

\section{2}

\section{Bedürfnis nach Geborgenheit und Identität}

Wenn Kultur Identität vermittelt, bestehen gute Chancen für die Stabilität dieser Kultur. Dazu muß eigentlich nicht viel gesagt werden, denn Entwicklung findet im kulturellen Kontext statt, und die Identitätsentwicklung erfolgt durch kulturelle Werte, die in der Sozialisation vermittelt und internalisiert worden sind.

Hier gehen wir im Sinne der Bindungstheorie (Bowlby 1969; Ainsworth et al. 1978) zunächst einmal davon aus, dass Menschen ein Bedürfnis nach Sicherheit und Geborgenheit haben, das ihnen in der frühesten Kindheit durch entsprechende emotionale Verfügbarkeit durch die primären Bezugspersonen vermittelt wird. Frühe Bindungserfahrungen wirken sich auf die weitere emotionale und kognitive Entwicklung und damit auch auf das Selbstkonzept aus. Solche frühen Erfahrungen sind über die Lebensspanne wirksam (Sroufe 1996).

Empirische Untersuchungen zur Bedeutung von Bindungssicherheit zeigen, dass keineswegs nur die frühen Bezugspersonen, sondern auch später die Peers und Partner in engen Beziehungen die Qualität der Bindung beeinflussen können (vgl. Hazan \& Shaver 1987). Für unser Thema soll im folgenden nur auf zwei Aspekte des Sicherheits- und Geborgenheitsbedürfnisses im Zusammenhang mit der Identitätsentwicklung eingegangen werden:

(a) Die Ausbildung der Identität zu einem ,independenten“ oder einem ,interdependenten“ Selbst (vgl. Markus \& Kitayama 1991) hat sich als sehr fruchtbar für die Erfassung von Kulturbesonderheiten in der Persönlichkeitsentwicklung erwiesen. Beim ,independenten“ Selbst werden Ziele der Unabhängigkeit und Autonomie vermittelt, die in entsprechendes Handeln einfließen und das Identitätserleben bestimmen. Beim ,interdependenten“ Selbst erlebt sich die Person 
als verbunden mit anderen Personen. Diese anderen Personen sind sozusagen Teil des eigenen Selbst. Die Überlappung und Verknüpfung zwischen eigenem Selbst und anderen Personen erlaubt nicht mehr, von dem ,eigenen“ Selbst zu sprechen. Interdependent ist in diesem Fall eine Integration von „eigenen“ Bedürfnissen und Erwartungen anderer Personen, bzw. den Mitgliedern der eigenen Gruppe, also der Familie, aber auch der Schule und später des Betriebes. Die Unterscheidung zwischen ,,independentem" und, interdependentem“ Selbst hat sich in empirischen Studien bereits beim Vergleich japanischer und deutscher Kinder nachweisen lassen (vgl. Kobayashi 1995).

(b) Hier ist jedoch noch ein anderer Aspekt der kulturvermittelten Identität relevant. Die These ist, dass eigene Identität immer auch kulturelle Identität ist. Diese These ist nicht besonders originell, wenn wir an Tajfels (1978) Arbeiten zur sozialen Identität denken. Danach entsteht Identität - einfach gesagt - im sozialen Kontext durch Vergleiche mit anderen und durch Abgrenzung von anderen. Die soziale Identität wird mit positiven Merkmalen versehen und führt zu einer Akzentuierung von Merkmaten, die in der eigenen sozialēn Gruppe erwünseht sind. Điese Akzentuierung hat jedoch weitere Abgrenżüngen gegenüber anderen Kulturgruppen zur Folge. Über diese soziale Identität hinaus kann auch eine ökologisch vermittelte Identität entstehen, die an Räume; Plătze; Orte, also an physikalisch definierbare, aber darüber hinaus mit bestimmten emotionalen Erlebnissen assoziierte ökologische Kontexte verbunden ist. Der physische Ort mit seinen identitätsrelevanten Erinnerungen vermittelt Vertrautheit und damit auch erlebte Zugehörigkeit und Geborgenheit. Insofern sind „Heimatgefühle" identitätsstiftend bzw. Ausdruck von Identität. Solche Heimatgefühle können ausgelöst werden durch Wiederbegegnung mit den Orten, in denen man aufgewachsen ist sowie auch durch Kontakte mit Personen aus eben diesen Orten. So sind identitätsrelevante und Geborgenheit stiftende Kontexte durch lokale Besonderheiten, durch Personen, durch Sprache und Interaktionsstile gekennzeichnet, kurz durch das, was in ihrer Substanz auch in Kultur eingeht.

Kulturelle Identität umfaßt qualitativ jedoch mehr als soziale und ökologische Identität, also mehr als eine durch Intergruppenvergleiche aufgebaute soziale Identität und mehr als ein lokal begrenzbares und emotional bedeutsames, Geborgenhëit assozijerendes HeimatgefühI. Wenn wir an die Aspekte denken, die wir bisher als psychologisch relevante Merkmale von Kultur beschrieben haben: Vermittlung von Deutungsschemata, Sinngebung, Kontrolle und Handlungsziele sowie Vermittlung von Geborgenheit, so müßten auch in kultureller Identität zumindest diese Aspekte verankert sein. 
Über die anfangs dargelegten Überlegungen zur Entstehung von Kultur hinaus soll daher im folgenden festgehalten werden, dass die Entwicklung von Kulturen eine individuelle Bindung an die Kultur voraussetzt, eine Identifikation mit den kulturellen Besonderheiten und damit verbunden eine Bereitschaft, die kulturellen Merkmale zu internalisieren und an die Angehörigen der eigenen Kultur weiterzugeben. Diese psychologische Voraussetzung für das Bestehen von Kultur müßte in der Ausbildung von kultureller Identität der Kulturangehörigen verankert sein.

Wie entsteht kulturelle Identität? Aus entwicklungs- und sozialpsychologischer Sicht erfolgt dıe İdentitätsentwïťcklung im Prozeß der Einbindung in eine soziale Einheit. Das individuelle personale Selbst ist immer auch ein soziales Selbst, sowohl im Selbsterleben als auch aus der Sicht von anderen. Selbstentwicklung erfolgt durch Differenzierung von anderen Personen; soziale Identität entsteht durch Differenzierung von sozialen Gruppen, und kulturelle Identität wird durch Differenzierung von anderen kulturellen Gruppen aufgebaut. Je unähnlicher die Anderen, desto eher bietet sich eine abgrenzbare Selbstdefinition an. Dieser Andere mag aus Sicht des Kulturangehörigen in Konkurrenz um knappe Ressourcen stehen oder die eigene Kultur physisch oder moralisch (z.B. durch andere Verhaltensregeln im sozialen Umgang oder im Umgang mit der Natur, mit den Göttern etc.) angreifen. Bei Bedrohung durch andere steigt das Bedürfnis der Sicherung der eigenen sozialen und kulturellen Identität. Auch hier wird durch soziale Prozesse die kulturelle Identität verstärkt. Dies erfolgt z.B. durch das Handeln des Gruppenführers, der damit seine eigene Position festigt, oder durch feindselige Handlungen gegenüber der Fremdgruppe, was die innere Solidarität und Zusammengehörigkeit stärkt.

Die emotionale Bindung des Selbst an die „Eigenkultur“ erfolgtdurch Abgrenzung von anđeren und Tdentifikation mit der eigenen Kultur. Die.Entwick. lung dieser-Identifikation und der kulturellen Identität erfolgt im Sozialisationsprozeß und duretrEernerfahrungenirn xerschiedenen Kontexten, wobe die in der ,culturat niche“ (Súper \& Harkness 1986) vermittelten Werte und emotionalen Bedingungen grundlëgende Dispositionen tormieren.

Es stellt sich abschließend die Frage, welche Formen kultureller Identität sich durch Kulturbegegnung im Sinne des Modells von Berry (1990) herausbilden können. Werden durch Globalisierung spezifische kulturelle Identitäten, die konkret sozial und lokal verankert sind, an Bedeutung verlieren, indem eine Vermischung und Integration verschiedenster Kulturen im Sinne einer „Weltkultur" erfolgt? Oder wird mit der Globalisierung eine „Einheitskultur“ aufgrund von kulturellem Imperialismus oder kultureller Integration und Assimila- 
tion geschaffen? Oder werden durch Globalisierung viele sozial und lokal verankerte Kulturen als abgegrenzte Einheiten in ihren Besonderheiten erstarkt? Diese Fragen sind wohl erst zu beantworten, wenn die Bedeutung von kultureller Identität und der Funktion von kulturellen Unterschieden sowohl für das Individuum als auch die Gesellschaft erkannt sind. Dabei wird man die Rolle eines individuellen Bedürfnisses nach kultureller Identität nicht übersehen können.

\section{Ausblick}

Eine Globalisierung ist unaufhaltsam. Sie ergibt sich aus den wirtschaftlichen Verflechtungen aller Regionen der ganzen Welt, und sie ist u.a. eine Folge der internationalen Beziehungen, der modernen Informationssysteme und der global operierenden Wirtschaftsunternehmen, die aufgrund des international vernetzten Kapitalmarktes untereinander verflochten sind.

Globalisienung ist erforderlich, weil weltweite Probleme globalen Ausmaßes zu bewältigen sind, Probleme, die nicht mehr nur eine Gesellschaft oder Kultur betreffen und auch nicht mehr aus der Sicht nur einer Gesellschaft gelöst werden können. Solche globalen Probleme ergeben sich u.a. aus massiven Veränderungen der ökologischen und klimatischen Bedingungen und dem enormen Anwachsen der Weltbevölkerung mit den dadurch bedingten Migrationsschüben. Die durch wirtschaftlichen, politischen und demographischen Druck ausgelösten Probleme globalen Ausmaßes bedürfen einer Lösung durch supranationale, globale Institutionen. Dafür sind internationale Rechtsordnungen und politische Organisationen erforderlich, die über die Bedürfnisse und Ressourcen von einzelnen Nationen hinaus handeln können.

Jedoch bedeutet die Lösung globaler Umbrüche durch globale Institutionen nicht, dass kulturelle Einheiten obsolet werden. Im Gegenteil, aus dem zuletzt Gesagten müssen wir schließen, dass erst die Bindung an kulturelle Einheiten mit ihren spezifischen kulturellen Besonderheiten (wie immer auch ihre regionalen und ethnischen o.a. Grenzen definiert sind), kulturelle und individuelle Identität schafft und damit auch Voraussetzungen für die Wahl von Handlungszielen und deren Realisierung im gegebenen kulturellen Werterahmen vermittelt.

Auch wenn die gegenwärtig beobachtbaren Globalisierungstendenzen zu einer Belebung von romantisch-intellektuellen Visionen einer "Weltkultur“ führen mögen - eine Entwicklung in Richtung auf eine globale, internationale Kultur erscheint psychologisch schwer begründbar. 


\section{Literatur}

Ainsworth, J., Blehar, M., Waters, E. \& Wall, S. (1978): Patterns of attachment. Hillsdale, NJ: Erlbaum

Berry, J.W. (1990): Psychology of acculturation: Understanding individual moving between cultures. In: Brislin, R.W.: Applied cross-cultural psychology. pp. 232-253. London: Sage

Bowlby, J. (1969): Attachment and loss: Vol. 1. Attachment. New York: Basic Books

Bronfenbrenner, U. (1979): The ecology of human development. Experiment by nature and design. London: Harvard University Press

Etzioni, A. (1994): The spirit of community: The reinvention of American society. New York: Simon \& Schuster

Friedlmeier, W. (1995): Subjekte Erziehungstheorien im Kulturvergleich. In: Trommsdorff, G. (Hg.): Kindheit und Jugend im Kulturvergleich, S. 43-64. Weinheim: Juventa

Friedman, J. (1994): Cultural identity and global process. London: Sage

Friedrichs, J. (1997): Globalisierung - Begriff und grundlegende Annahmen (Globalization - Concept and basic asumptions). Politik und Zeitgeschichte, B33-34/97, S. 3-11

Hazan, C. \& Shaver, P.R. (1987): Romantic love conceptualized as an attachment process. Journal of Personality and Social Psychology, 52, pp. 511-524

Heberer, T. (1997): Ostasien und der Westen: Globalisierung oder Regionalismus? (East of Asia and the West: Globalization or regionalization?). ASIEN, 63, S. 5-53

Hofstede, G. (Ed.) (1980): Culture's consequences. International differences in work-related values. London: Sage

Huntington, S.P. (1996): The clash of civilizations and the remaking of world order. New York: Simon \& Schuster

Kobayashi, M. (1995): Selbstkonzept und Empathie im Kulturvergleich - Ein Vergleich deutscher und japanischer Kinder. Konstanz: Universitätsverlag

Kornadt, H.-J. (1986): Internationales Marketing aus der Sicht kulturvergleichender Psychologie (International marketing in perspective of cross-cultural psychology). Werbeforschung und Praxis, 35, S. 97-104

Markus, H.R. \& Kitayama, S. (1991): Culture and the self: Implications for cognition, emotion and motivation. Psychological Review, 98, pp. 224-253

Mishima, K. (1993): Das Zusammenspiel von kollektiver und individueller Identität als Merkmal japanischer Kultur (Interaction of collective and individual identity as characteristic of Japanese culture). In: Kornadt, H.-J. \& Trommsdorff, G. (Hg.) (1993): Deutsch-japanische Begegnungen in den Sozialwissenschaften. Wiederbeginn wissenschaftlicher Kooperation in gesellschaftsbezogener Forschung. pp. 137-148. Konstanz: Universitätsverlag

Piaget, J. (1992): Das Weltbild des Kindes (La representation du monde chez l'enfant). München: DTV. (Original work published 1926)

Ritzer, G. (1993): The McDonaldization of society: An investigation into the changing character of contemporary social life. Thousand Oaks: Pine Forge Press 
Rohner, R.P. \& Pettengill, S.M. (1985): Perceived parental acceptance-rejection and parental control among Korean adolescents. Special Issue: Family development. Child Development, 56, pp. 524-528

Sroufe, L.A. (1996): Emotional development. The organization of emotional life in the early years. Cambridge: Cambridge University Press

Super, C.M. \& Harkness, S. (1986): The developmental niche: A conceptualization at the interface of child and culture. International Journal of Behavioral Development, 9 , pp. 545-569

Suzuki, T. (1993): Japan auf dem Weg ins Jahr 2000 (Japan on the way to the year 2000). In: Kornadt, H.-J. \& Trommsdorff, G. (Hg.)(1993): Deutsch-japanische Begegnungen in den Sozialwissenschaften. Wiederbeginn wissenschaftlicher Kooperation in gesellschaftsbezogener Forschung . S. 195-206. Konstanz: Universitätsverlag

Tajfel, H. (1978): Intergroup behaviour: II. Group perspectives. In Tajfel, H. \& Fraser, C. (Eds.): Introducing social psychology: An analysis of individual reaction and response. pp. 423-446. Middlesex (England): Penguin Books

Triandis, H.C. (1995): Individualism and collectivism. Boulder: Westview Publishers

Trommsdorff, G. (1985): Some comparative aspects of socialization in Japan and Germany. In: Lagunes, I.R. \& Poortinga, Y.H. (Eds.): From a different perspective: Studies of behavior across cultures. pp. 231-240. Amsterdam: Swets \& Zeitlinger

Trommsdorff, G. (1995): Parent-adolescent relations in changing societies: A crosscultural study. In: Noack, P., Hofer, M. \& Youniss, J. (Eds.): Psychological responses to social change: Human development in changing environments. pp. 189-218. Berlin: DeGruyter

Trommsdorff, G. (1996): Werte und Wertewandel im kulturellen Kontext aus psycho logischer Sicht (Values and value change in cross-cultural context in psychological perspective). In: Janssen, E., Möhwald, U. \& Ölschleger, H.D. (Hg.): Gesellschaften im Umbruch? Aspekte des Wertewandels in Deutschland, Japan und Osteuropa. Monographien des Deutschen Instituts für Japanforschung der Phillipp-Franz-vonSiebold-Stiftung, Band 15, pp. 13-40. München: Iudicium

Trommsdorff, G. (1998a) (in press-a): Autonomie und Verbundenheit im kulturellen Vergleich von Sozialisationsbedingungen (Autonomy and relatedness in crosscultural comparison of conditions of socialization). In: Leu, H.R. \& Krappmann, L. (Hg.): Zwischen Autonomie und Verbundenheit. Frankfurt: Suhrkamp

Trommsdorff, G. (1998b) (in press-b): Social an psychological aspects of ongoing changes in Japan: Introduction . In: Trommsdorff, G., Friedlmeier, W. \& Kornadt, H.-J. (Eds.): Japan in transition - A comparative view on social and psychological aspects. Lengerich: Pabst Science Publishers

Trommsdorff, G. (1998c) (in press-c): Social change and individual development: The Role of subjective belief. In: Silbereisen, R. \& Bynner, J. (Eds.): Adversity and challenge in life in the new Germany and England. Hampshire, England: The MacMillan Press Ltd.

Trommsdorff, G., Suzuki, T. \& Sasaki, M. (1990): Soziale Ungleichheiten in Japan und der Bundesrepublik Deutschland (Social inequalities in Japan and the Federal Republic of Germany). In: Hayashi, C. \& Suzuki, T. (Eds.): Beyond Japanese social values: Trends and cross-national perspectives. pp. 427-450. Tokyo: The Institute of 
Statistical Mathematics. (Abdruck des Aufsatzes aus Kölner Zeitschrift für Soziologie und Sozialpsychologie, 1987, 39, 471-495)

Whiting, B.B. \& Whiting, J.W.M. (1975): Children of six cultures: A psycho-cultural analysis. Cambridge, MA: Harvard University Press 